\title{
Borreliacidal activity of saliva of the tick Amblyomma americanum
}

\author{
K. E. LEDIN ${ }^{1}$, N.S. ZEIDNER ${ }^{1}$, J. M. C. RIBEIRO ${ }^{2}$, B. J. BIGGERSTAFF ${ }^{1}$, \\ M. C. DOLAN ${ }^{1}$, G. DIETRICH ${ }^{1}$, L. VREDEVOE ${ }^{3}$ and J. PIESMAN ${ }^{1}$ \\ ${ }^{1}$ Division of Vector-Borne Infectious Diseases, National Center for Infectious Diseases, Centers for Disease Control and \\ Prevention, Fort Collins, Colorado, U.S.A., ${ }^{2}$ Laboratory of Malaria and Vector Research, Section of Vector Biology, National \\ Institute of Allergy and Infectious Diseases, National Institutes of Health, Bethesda, Maryland, U.S.A. and ${ }^{3}$ Biological \\ Sciences Department, California Polytechnic State University, San Luis Obispo, California, U.S.A.
}

\begin{abstract}
Amblyomma americanum (Linneaus) (Acari: Ixodidae), an important tick vector of human and animal disease, is not a competent vector of the bacterial agent of Lyme disease, Borrelia burgdorferi, although its range overlaps the geographical distribution of Lyme disease within the United States. A possible mechanism that could prevent acquisition of $B$. burgdorferi spirochetes from infected hosts is the toxic effect of $A$. americanum saliva on $B$. burgdorferi. The data presented here indicate that after 24 and $48 \mathrm{~h}$ of exposure to A. americanum saliva, significantly fewer $B$. burgdorferi were alive compared to treatment controls as assessed by spirochete motility under dark-field microscopy and resistance to the dead stain, propidium iodide. After $48 \mathrm{~h}$, fewer than $13 \%$ of saliva-exposed B. burgdorferi were alive. In contrast, significantly more B. burgdorferi exposed to Ixodes scapularis (Acari: Ixodidae) saliva survived after 24 or $48 \mathrm{~h}$ compared to A. americanum saliva or treatment controls.
\end{abstract}

Key words. Amblyomma americanum, Ixodes scapularis, borreliacidal effect, salivary glands, salivation, tick saliva.

\section{Introduction}

Amblyomma americanum (Linnaeus), the lone star tick, is the primary vector of Ehrlichia chaffeensis, the bacterial agent of human monocytic ehrlichiosis (Anderson et al., 1993). This aggressive human-biting tick species is also thought to vector other potential pathogens including Ehrlichia ewingii, Rickettsia rickettsii, R. amblyommii, Borrelia lonestari, Francisella tularensis and Coxiella burnetii (Calhoun, 1954; Burgdorfer, 1975; Barbour etal., 1996; Murphy et al., 1998; Burkot et al., 2001; Childs \& Paddock, 2003). Amblyomma americanum ticks feed for 4-14 days (Sauer \& Hair, 1972) on a wide variety of mammalian, reptilian and avian hosts (Cooley \& Kohls, 1944; Clymer

Correspondence: Nordin S. Zeidner, Division of Vector-Borne Infectious Diseases, Centers for Disease Control and Prevention, PO Box 2087, Fort Collins, CO 80522, U.S.A. Tel.: +1970 221 6495; fax: +1 9702254257 ; e-mail: naz2@cdc.gov etal., 1970). Some reservoir hosts are also commonly infested by Ixodes scapularis Say, the tick vector of Borrelia burgdorferi, the agent of Lyme disease in the United States (Magnarelli etal., 1986; Oliver etal., 1999; Clark etal., 2001). Amblyomma americanum is found throughout geographical regions that overlap areas of human Lyme disease occurrence, perennially in southern latitudes and seasonally in northern latitudes (Bishopp \& Trembley, 1945; Anderson \& Magnarelli, 1980; Hair \& Bowman, 1986; Ginsberg et al., 1991; Luckhart etal., 1991; Keirans \& Lacombe, 1998). Despite the confluence in geographical distribution and host species, there is no evidence that $A$. americanum transmits B. burgdorferi (Piesman \& Happ, 1997).

During A. americanum, I. scapularis and other Ixodid tick feeding, saliva is continuously secreted into the host (McMullen \& Sauer, 1978; Coons et al., 1986; Sonenshine, 1991). Ixodid saliva is known to have a broad range of haemostatic and immunomodulatory effects in mammalian hosts (Ribeiro, 1995; Wikel, 1999). Anecdotal reports indicate significant inflammation and irritation at the site of 
A. americanum feeding on humans (Armstrong et al., 2001), and evidence for a haemolytic saliva factor (Zhu et al., 1997) suggests that $A$. americanum saliva could have a broadly cytotoxic effect.

Arthropod vector refractoriness to a pathogen can be classified into three categories: (1) a lack of acquisition; (2) an inability to maintain growth of the pathogen; or (3) an inability to transmit the pathogen. Spirochetes have been detected in $0.2-15.6 \%$ of field-collected $A$. americanum (Magnarelli etal., 1986; Schulze etal., 1986; Levine et al., 1991; Luckhart etal., 1991; Teltow et al., 1991; Luckhart etal., 1992; Feir etal., 1994; Rawlings \& Teltow, 1994; Sonenshine etal., 1995; Ouellette et al., 1997; Stromdahl et al., 2001). However, many of the reports of spirochetepositive $A$. americanum predate the discovery of the spirochete $B$. lonestari, or use methods that would not be able to discriminate between the two spirochete species, B. burgdorferi and B. lonestari (Barbour et al., 1996). Although as many as $25 \%$ of experimentally exposed $A$. americanum larvae acquired spirochetes during infectious feedings, nearly all of these larvae were spirochete-negative before moulting to the nymphal stage (Piesman \& Sinsky, 1988; Mather \& Mather, 1990; Ryder etal., 1992; Piesman \& Happ, 1997). There is only one report of trans-stadial maintenance of infection in $A$. americanum nymphs (Ryder etal., 1992) and one report of spirochete isolates from three pools of A. americanum adults (Teltow et al., 1991). Moreover, there has been no successful transmission of B. burgdorferi between infected and naïve hosts by A. americanum (Mukolwe et al., 1992; Ryder et al., 1992; Oliver et al., 1993; Sanders \& Oliver, 1995; Piesman \& Happ, 1997). It was hypothesized that a cytotoxic effect of $A$. americanum saliva might be one mechanism preventing the acquisition of $B$. burgdorferi spirochetes from an infected reservoir host. In the current study, the survival of $B$. burgdorferi exposed to $A$. americanum saliva was measured. To document that this is a species-specific effect in a refractory vector, the survival of B. burgdorferi exposed to saliva of $I$. scapularis was also measured.

\section{Materials and methods}

\section{Tick infestation and saliva collection}

Colony-produced, pathogen-free $A$. americanum adults were purchased from the Oklahoma State University Tick Rearing Facility (Stillwater, Oklahoma). Colony-maintained I. scapularis adults originated from the Lyme Disease Vector Laboratory of the CDC (Fort Collins, Colorado). Adult female New Zealand rabbits (Western Oregon Rabbit Co., Philomath, Oregon) were infested with 20-30 pairs of A. americanum or I. scapularis adults, as described previously (Piesman etal., 1991). During infestation, ticks were confined within fabric bags completely enclosing each ear of the rabbit. Tick engorgement was monitored daily, and adult females were removed from rabbit ears at the beginning of the rapid engorgement phase, typically 11-
13 days post-infestation for $A$. americanum or 5-7 days post-infestation for I. scapularis. All animal procedures were reviewed and approved by the CDC Institutional Animal Care and Use Committee (AUP no. 00-08-010 RAB).

Near-replete ticks were prepared for saliva collection as described previously (Ribeiro et al., 2004). Briefly, each immobilized tick had its mouthparts fitted with a finely drawn capillary tube and $5 \mu \mathrm{L}$ of $5 \%$ pilocarpine in methanol was applied topically to the dorsal scutum. Salivating ticks were maintained for $1-2 \mathrm{~h}$ in a humid chamber at $35^{\circ} \mathrm{C}$; collected saliva was pooled on ice and stored at $-70^{\circ} \mathrm{C}$ until use. One $\mu \mathrm{L}$ aliquots of saliva from each pool were tested by an HPLC-MS/MS method to determine a pg to $\mathrm{mg}$ range of pilocarpine concentrations (Ribeiro et al., 2004) and this value was utilized to determine control standards for subsequent borreliacidal assays.

\section{Spirochete culture}

Frozen stocks of low-passage B31 B. burgdorferi isolates (Shelter Island, New York) were reconstituted in BSK-H culture medium and maintained at $35^{\circ} \mathrm{C}$ to log phase (Piesman, 1993). Spirochete cultures were then diluted with BSK-H to a density of $4.7 \times 10^{7}$ viable spirochetes per $\mathrm{mL}$ as counted in a Petroff-Hauser chamber under dark-field microscopy, and distributed in $15 \mu \mathrm{L}$ aliquots into $0.7 \mathrm{~mL}$ tubes for a total of $7.05 \times 10^{5}$ spirochetes per tube. A mean starting concentration of 155 live spirochetes per field was assessed prior to the addition of specific treatments. Each $15 \mu \mathrm{L}$ aliquot of spirochetes in BSK-H was then treated with either $15 \mu \mathrm{L}$ of sterile PBS, $15 \mu \mathrm{L}$ of $1.33 \mathrm{mg} / \mathrm{mL}$ pilocarpine (based on the mid-range of pilocarpine found in previous analysis of tick saliva, Ribeiro et al., 2004) in sterile PBS, or $15 \mu \mathrm{L}$ of tick saliva, and incubated at $35^{\circ} \mathrm{C}$. A total of 10 cultures were prepared with each treatment (PBS, pilocarpine control, tick saliva) for each tick species; $10 \mu \mathrm{L}$ samples taken from each culture were then assessed for spirochete survival at 0,24 and $48 \mathrm{~h}$. Time points for spirochete survival assessment were chosen based on previous observations of post-feeding decreases in spirochete-positive ticks (Ryder et al., 1992).

\section{Spirochete survival assessment}

Spirochete survival was evaluated by enumeration of spirochetes stained with the live/dead BacLight Viability Kit (Molecular Probes, Eugene, Oregon). Briefly, fluorescence stains SYTO 9 (live stain) and propidium iodide (dead stain) were combined at a 1:100 dilution in sterile PBS (Invitrogen, Grand Island, New York). A total of $0.5 \mu \mathrm{L}$ of this solution was then mixed with $10 \mu \mathrm{L}$ from each spirochete culture. This staining concentration was chosen in preliminary experiments for optimal discrimination of spirochetes using fluorescent microscopy. Stained cultures were suspended on a standard glass slide under a $22 \times 22 \mathrm{~mm}$ coverslip and viewed at $100 \times$ magnification 
through FITC and rhodamine filters. Live and dead spirochetes were counted from 10 randomly chosen high-power fields per slide.

\section{Statistics}

Mean counts of live spirochetes made at 24 and $48 \mathrm{~h}$ were compared using an over-dispersed Poisson generalized linear mixed model (Wolfinger \& O’Connell, 1993). Fixed effects for time and treatment (A. americanum saliva, I. scapularis saliva, pilocarpine, and PBS) and their interaction were included, as were the random effects of separate trials and trial by time interaction, to accommodate potentially differential effects with each trial. Models were compared using the likelihood ratio chi-squared test to evaluate both fixed effects and covariance parameters for statistical significance, with $P<0.05$ considered statistically significant. Final comparisons of treatment effects were made using the parsimonious model thus derived and computations were performed using the glimmix macro in SAS v9.1 (SAS Institute, Cary, North Carolina).

\section{Results and discussion}

To evaluate the effect of tick saliva from $I$. scapularis and A. americanum on B. burgdorferi, a fluorescence method was used to quantify spirochete viability. With this method, live spirochetes, staining green (Figs 1A and B), could be readily visualized and distinguished from injured and dead spirochetes, which stained red (Figs $1 \mathrm{C}$ and D). The uptake of live and dead stains observed after saliva or control treatments of spirochete cultures was consistent with the preliminary dark-field microscopy observations of treatment-induced differences in spirochete appearance and motility. It was not possible to quantify the total number of dead spirochetes at 24 and $48 \mathrm{~h}$ consistently due to disintegration of cells in more severe treatments (data not shown); therefore, only live spirochete numbers were used for analysis. This analysis (Fig. 2) indicates that treatment with $A$. americanum saliva significantly reduced the average number of live spirochetes at both $24 \mathrm{~h}($ mean $=39.6)$ and $48 \mathrm{~h}($ mean $=20.1)$ compared with both pilocarpine at 24 and $48 \mathrm{~h}(82.1 / 58.2)$ and PBS at 24 and $48 \mathrm{~h}$ (133/112) (all $P<0.001)$. Furthermore, average counts for $A$. americanum saliva were also significantly lower than average counts for I. scapularis treatment at 24 and $48 \mathrm{~h}(100.2 / 75.0, P<0.001$ for both time points). In contrast, I. scapularis did not yield significantly different spirochete counts than pilocarpine at both time points, while demonstrating a significant killing effect compared to PBS at 24 and $48 \mathrm{~h}(P<0.001$ for both). Finally, differences in the borreliacidal activity of A. americanum and I. scapularis saliva could not be accounted for by differences in $\mathrm{pH}$ after saliva preparation; both tick salivas were basic, with $\mathrm{pH}$ measuring between 9.5 and 10.0 (data not shown).

Numerous vector-competency studies have demonstrated that $A$. americanum is unable to maintain or transmit $B$. burgdorferi. Although $A$. americanum can acquire

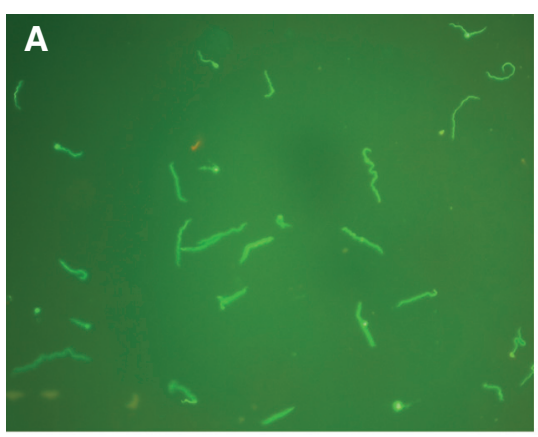

PBS

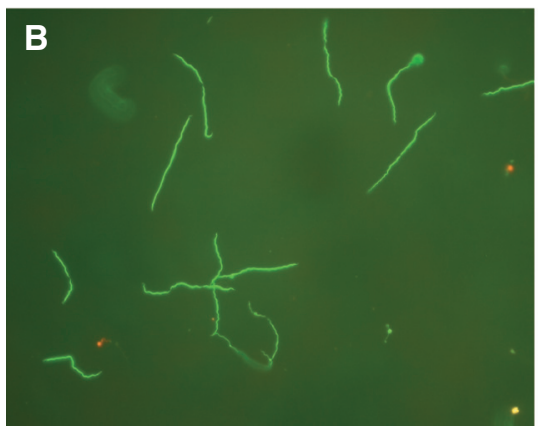

I. scapularis saliva

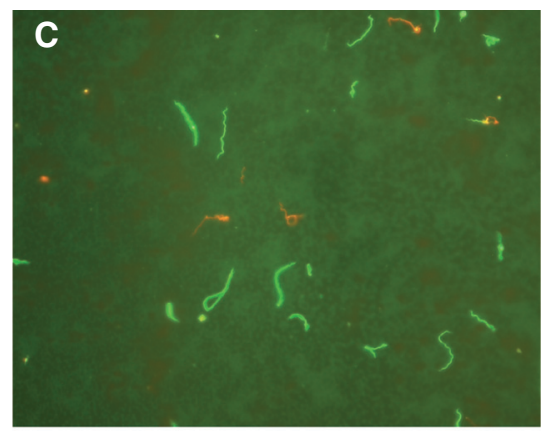

Pilocarpine

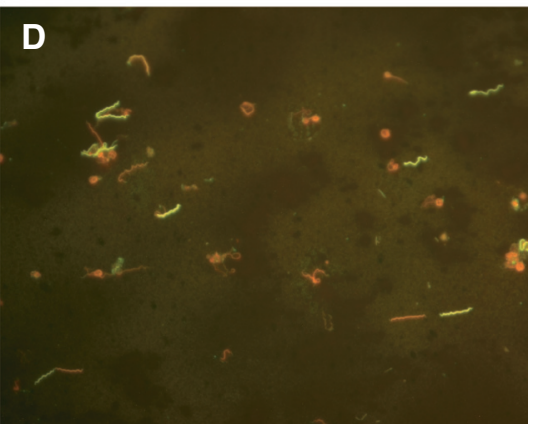

A. americanum saliva
Fig. 1. Fluorescent microscopy indicating live (SYTO9, green) and dead (propidium iodide, red) staining as a measure of $B$. burdgorferi survival $48 \mathrm{~h}$ after exposure to specific in vitro treatments $(100 \times)$ as described in the Materials and Methods. (A) PBS, (B) I. scapularis saliva, (C) Pilocarpine, (D) A. americanum saliva. 


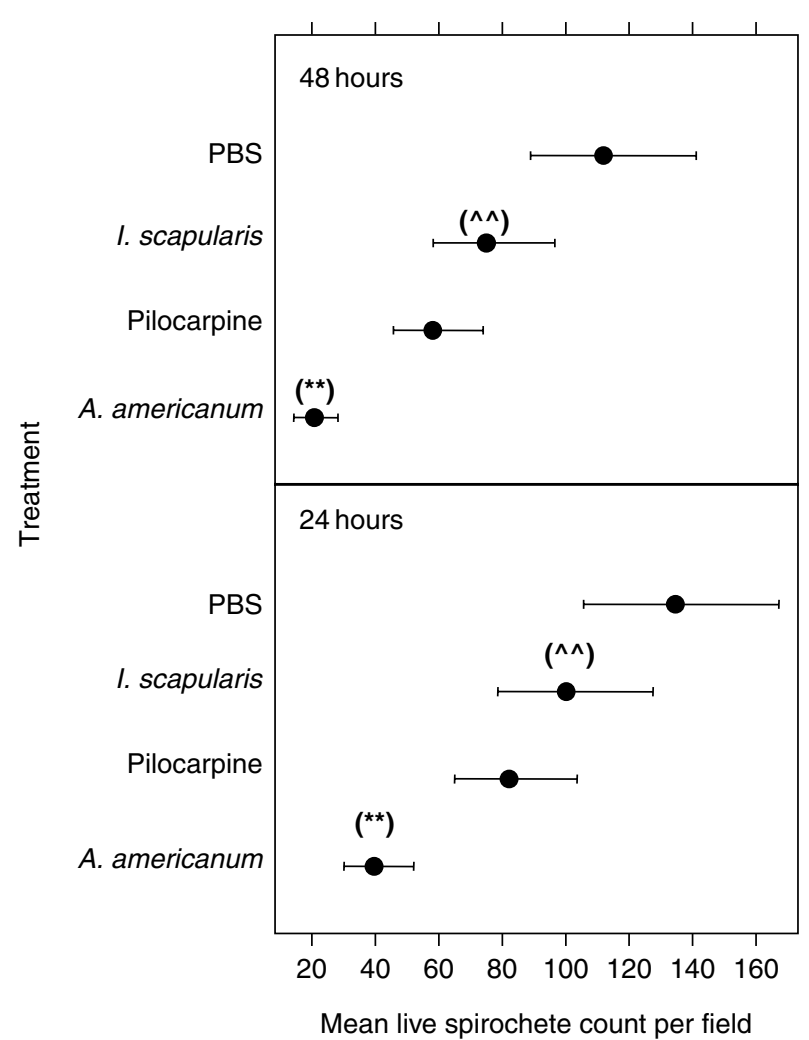

Fig. 2. (A) Mean live B. burgdorferi counts per 10 high powered $(100 \times)$ fields and estimated $95 \%$ confidence intervals after exposure to A. americanum saliva, pilocarpine, I. scapularis saliva, or PBS at 24 and $48 \mathrm{~h}$ post-exposure. Means were computed using the model as described in the Materials and Methods. Error bars indicate the computed confidence intervals. ${ }^{* *}$ and ${ }^{\wedge}$ indicate a significant difference in viable spirochete numbers when comparing A. americanum saliva to pilocarpine controls and to I. scapularis saliva $(P<0.001$, for both, Chi-squared test). Note the confidence intervals are asymmetric due to the logarithmic nature of the Poisson model used.

B. burgdorferi by experimental feeding, the number of spirochetes detectable in $A$. americanum rapidly decreases post-feeding, as 4.6-fold fewer $A$. americanum than I. scapularis larvae are spirochete-positive $48 \mathrm{~h}$ after taking an infective bloodmeal (Ryder et al., 1992). The number of A. americanum larvae with detectable spirochetes decreases to zero within 10 days post-feeding, and remains so for the duration of the larval stage (Piesman \& Sinsky, 1988). The results of the studies presented here suggest that the significant borreliacidal activity of $A$. americanum saliva may be a possible mechanism for the lack of acquisition of B. burgdorferi and transmission in vivo. The cytotoxic effect of $A$. americanum saliva in reducing spirochete numbers (Fig. 2) could readily reduce the dose of spirochetes acquired during feeding on a highly spirochetemic host. Likewise, saliva injected into the host by feeding A. americanum ticks (McMullen \& Sauer, 1978) might reduce spirochete numbers at the cutaneous bite site. Moreover, saliva re-ingested with host fluids could gradu- ally cause spirochete mortality as the bloodmeal is processed within the tick midgut (Coons et al., 1986). Why $A$. americanum saliva would have a cytotoxic effect on the spirochete B. burgdorferi and not a Borrelia spp. associated with A. americanum, B. lonestari (Barbour et al., 1996) needs to be investigated further. With the advent of new techniques to culture B. lonestari (Varela et al., 2004) these comparative studies can now be attempted.

The effect of A. americanum saliva on B. burgdorferi spirochetes is in significant contrast to the effect of I. scapularis saliva (Figs 1B and 2). Ixodes scapularis saliva did not have a borreliacidal effect greater than the pilocarpine control treatment, and appears not to be an obstacle in maintaining I. scapularis vector competency. In addition to the anticoagulation and immunomodulatory activities of I. scapularis saliva in the mammalian host (Ribeiro et al., 1985), studies have suggested the presence of a factor in I. scapularis saliva that is permissive for enhancement of B. burgdorferi dissemination in vivo (Zeidner et al., 2002). It has been hypothesized that cofeeding of Dermacentor variabilis with I. scapularis can increase D. variabilis infection rates (Piesman \& Happ, 1997). Finally, viable B. burgdorferi have been collected directly from pilocarpine-stimulated collections of I. scapularis saliva (Ribeiro et al., 1987; Ewing et al., 1994).

The current study has demonstrated a cytotoxic effect of pilocarpine on B. burgdorferi. Pilocarpine is known to have a physiological effect on mammalian cells in quantities as low as $500 \mu \mathrm{m}$ (Arzt et al., 1989), and has been detected in saliva collected from $A$. americanum and I. scapularis (Ribeiro etal., 2004). Therefore, as noted by Ribeiro et al. (2004), the concentration of pilocarpine should be determined in every sample of saliva destined for in vitro testing on living cells, and compensated for with appropriate controls or by the addition of an atropine inhibitor (Arzt et al., 1989). In this study, A. americanum saliva had a significantly greater borreliacidal effect on spirochetes than exposure to both background levels of pilocarpine or I. scapularis saliva (Fig. 2). The difference in borreliacidal activity might be the result of a specific molecule or compound present in the saliva of $A$. americanum but not $I$. scapularis ticks. Proteins are abundant in the saliva of these two tick vectors (Madden et al., 2002; Valenzuela et al., 2002). A direct comparison of saliva from these vector species by SDS-PAGE gel revealed at least two protein differences in the 200-90 kDaA range and four distinct differences within the $34-20 \mathrm{kDaA}$ range (data not shown). Isolation and identification of a borreliacidal factor from A. americanum saliva could lead to the development of antispirochete compounds for prophylactic treatment of B. burgdorferi exposure, or to the molecular engineering of transgenic, B. burgdorferi-refractory I. scapularis ticks to study mechanisms of vector competency.

\section{Acknowledgements}

This work was supported in part by an American Society for Microbiology postdoctoral research fellowship. 


\section{References}

Anderson, J.F. \& Magnarelli, L.A. (1980) Vertebrate host relationships and distribution of ixodid ticks (Acari: Ixodidae) in Connecticut, USA. Journal of Medical Entomology, 17, 314-23.

Anderson, B.E., Sims, K.G., Olson, J.G., Childs, J.E., Piesman, J.F., Happ, C.M., Maupin, G.O. \& Johnson, B.J. (1993) Amblyomma americanum: a potential vector of human ehrlichiosis. American Journal of Tropical Medicine and Hygiene, 49, 239-44.

Armstrong, P.M., Brunet, L.R., Spielman, A. \& Telford, S.R. (2001) 3rd Risk of Lyme disease: perceptions of residents of a Lone Star tick-infested community. Bulletin of the World Health Organization, 79, 916-25.

Arzt, E.S., Fernandez-Castelo, S., Diaz, A., Finkielman, S. \& Nahmod, V.E. (1989) The muscarinic agonist pilocarpine inhibits DNA and interferon-gamma synthesis in peripheral blood mononuclear cells. International Journal of Immunopharmacology, 11, 275-81.

Barbour, A.G., Maupin, G.O., Teltow, G.J., Carter, C.J. \& Piesman, J. (1996) Identification of an uncultivable Borrelia species in the hard tick Amblyomma americanum: possible agent of a Lyme disease-like illness. Journal of Infectious Diseases, 173, 403-9.

Bishopp, F.C. \& Trembley, H.L. (1945) Distribution and hosts of certain North American ticks. Journal of Parasitology, 31, 1-54.

Burgdorfer, W. (1975) A review of Rocky Mountain spotted fever (tick-borne typhus), its agent, and its tick vectors in the United States. Journal of Medical Entomology, 12, 269-78.

Burkot, T.R., Mullen, G.R., Anderson, R., Schneider, B.S., Happ, C.M. \& Zeidner, N.S. (2001) Borrelia lonestari DNA in adult Amblyomma americanum ticks, Alabama. Emerging Infectious Diseases, 7, 471-3.

Calhoun, E.L. (1954) Natural occurrence of tularemia in the lone star tick, Amblyomma americanum (Linn.), and in dogs in Arkansas. American Journal of Tropical Medicine and Hygiene, 3, 360-6.

Childs, J.E. \& Paddock, C.D. (2003) The ascendancy of Amblyomma americanum as a vector of pathogens affecting humans in the United States. Annual Review of Entomology, 48, 307-37.

Clark, K.L., Oliver, J.H. Jr, Grego, J.M., James, A.M., Durden, L.A. \& Banks, C.W. (2001) Host associations of ticks parasitizing rodents at Borrelia burgdorferi enzootic sites in South Carolina. Journal of Parasitology, 87, 1379-86.

Clymer, B.C., Howell, D.E. \& Hair, J.A. (1970) Environmental alteration in recreational areas by mechanical and chemical treatment as a means of lone star tick control. Journal of Economic Entomology, 63, 504-9.

Cooley, R.A. \& Kohls, G.M. (1944) The genus Amblyomma (Ixodidae) in the United States. Journal of Parasitology, 30, 77-111.

Coons, L.B., Rosell-Davis, R. \& Tarnowksi, B.I. (1986) Bloodmeal digestion in ticks. Morphology, Physiology, and Behavioral Biology of Ticks (ed. by J. R. Sauer and J. A. Hair), pp. 248279. John Wiley \& Sons, New York.

Ewing, C., Scorpio, A., Nelson, D.R. \& Mather, T.N. (1994) Isolation of Borrelia burgdorferi from saliva of the tick vector, Ixodes scapularis. Journal of Clinical Microbiology, 32, 755-8.

Feir, D., Santanello, C.R., Li, B.W., Xie, C.S., Masters, E., Marconi, R. \& Weil, G. (1994) Evidence supporting the presence of Borrelia burgdorferi in Missouri. American Journal of Tropical Medicine and Hygiene, 51, 475-82.

Ginsberg, H.S., Ewing, C.P., O'Connell, A.F. Jr, Bosler, E.M., Daley, J.G. \& Sayre, M.W. (1991) Increased population densities of Amblyomma americanum (Acari: Ixodidae) on Long Island, New York. Journal of Parasitology, 77, 493-5.

Hair, J.A. \& Bowman, J.L. (1986) Behavioral ecology of Amblyomma americanum (L.). Morphology, Physiology, and Behavioral Biology of Ticks (ed. by J. R. Sauer and J. A. Hair), pp. 406-427. John Wiley \& Sons, New York.

Keirans, J.E. \& Lacombe, E.H. (1998) First records of Amblyomma americanum, Ixodes (Ixodes) dentatus, and Ixodes (Ceratixodes) uriae (Acari: Ixodidae) from Maine. Journal of Parasitology, 84, 629-31.

Levine, J.F., Sonenshine, D.E., Nicholson, W.L. \& Turner, R.T. (1991) Borrelia burgdorferi in ticks (Acari: Ixodidae) from coastal Virginia. Journal of Medical Entomology, 28, 668-74.

Luckhart, S., Mullen, G.R., Durden, L.A. \& Wright, J.C. (1992) Borrelia sp. in ticks recovered from white-tailed deer in Alabama. Journal of Wildife Diseases, 28, 449-52.

Luckhart, S., Mullen, G.R. \& Wright, J.C. (1991) Etiologic agent of Lyme disease, Borrelia burgdorferi, detected in ticks (Acari: Ixodidae) collected at a focus in Alabama. Journal of Medical Entomology, 28, 652-7.

Madden, R.D., Sauer, J.R. \& Dillwith, J.W. (2002) A proteomics approach to characterizing tick salivary secretions. Experimental and Applied Acarology, 28, 77-87.

Magnarelli, L.A., Anderson, J.F., Apperson, C.S., Fish, D., Johnson, R.C. \& Chappell, W.A. (1986) Spirochetes in ticks and antibodies to Borrelia burgdorferi in white-tailed deer from Connecticut, New York State, and North Carolina. Journal of Wildlife Diseases, 22, 178-88.

Mather, T.N. \& Mather, M.E. (1990) Intrinsic competence of three ixodid ticks (Acari) as vectors of the Lyme disease spirochete. Journal of Medical Entomology, 27, 646-50.

McMullen, H.L. \& Sauer, J.R. (1978) The relationship of phosphodiesterase and cyclic AMP to the process of fluid secretion in the salivary glands of the ixodid tick Amblyomma americanum. Experientia, 34, 1030-31.

Mukolwe, S.W., Kocan, A.A., Barker, R.W., Kocan, K.M. \& Murphy, G.L. (1992) Attempted transmission of Borrelia burgdorferi (Spirochaetales: Spirochaetaceae) (JDI strain) by Ixodes scapularis (Acari: Ixodidae), Dermacentor variabilis, and Amblyomma americanum. Journal of Medical Entomology, 29, 673-7.

Murphy, G.L., Ewing, S.A., Whitworth, L.C., Fox, J.C. \& Kocan, A.A. (1998) A molecular and serologic survey of Ehrlichia canis, E. chaffeensis, and E. ewingii in dogs and ticks from Oklahoma. Veterinary Parasitology, 79, 325-39.

Oliver, J.H. Jr, Chandler, F.W. Jr, Luttrell, M.P., James, A.M., Stallknecht, D.E., McGuire, B.S., Hutcheson, H.J., Cummins, G.A. \& Lane, R.S. (1993) Isolation and transmission of the Lyme disease spirochete from the southeastern United States. Proceedings of the National Academy of Sciences of the USA, 90, 7371-5.

Oliver, J.H. Jr, Magnarelli, L.A., Hutcheson, H.J. \& Anderson, J.F. (1999) Ticks and antibodies to Borrelia burgdorferi from mammals at Cape Hatteras, NC and Assateague Island, MD and VA. Journal of Medical Entomology, 36, 578-87.

Ouellette, J., Apperson, C.S., Howard, P., Evans, T.L. \& Levine, J.F. (1997) Tick-raccoon associations and the potential for Lyme disease spirochete transmission in the coastal plain of North Carolina. Journal of Wildlife Diseases, 33, 28-39.

Piesman, J. (1993) Standard system for infecting ticks (Acari: Ixodidae) with the Lyme disease spirochete, Borrelia burgdorferi. Journal of Medical Entomology, 30, 199-03.

Piesman, J. \& Happ, C.M. (1997) Ability of the Lyme disease spirochete Borrelia burgdorferi to infect rodents and three species 
of human-biting ticks (blacklegged tick, American dog tick, lone star tick) (Acari: Ixodidae). Journal of Medical Entomology, 34, 451-6.

Piesman, J., Maupin, G.O., Campos, E.G. \& Happ, C.M. (1991) Duration of adult female Ixodes dammini attachment and transmission of Borrelia burgdorferi, with description of a needle aspiration isolation method. Journal of Infectious Diseases, 163, 895-7.

Piesman, J. \& Sinsky, R.J. (1988) Ability of Ixodes scapularis, Dermacentor variabilis, and Amblyomma americanum (Acari: Ixodidae) to acquire, maintain, and transmit Lyme disease spirochetes (Borrelia burgdorferi). Journal of Medical Entomology, 25, 336-9.

Rawlings, J.A. \& Teltow, G.J. (1994) Prevalence of Borrelia (Spirochaetaceae) spirochetes in Texas ticks. Journal of Medical Entomology, 31, 297-301.

Ribeiro, J. (1995) How ticks make a living. Parasitology Today, 11, 91-93.

Ribeiro, J.M., Makoul, G.T., Levine, J., Robinson, D.R. \& Spielman, A. (1985) Antihemostatic, antiinflammatory, and immunosuppressive properties of the saliva of a tick, Ixodes dammini. Journal of Experimental Medicine, 161, 332-44.

Ribeiro, J., Mather, T.N., Piesman, J. \& Spielman, A. (1987) Dissemination and salivary delivery of Lyme disease spirochetes in vector ticks (Acari: Ixodidae). Journal of Medical Entomology, 24, 201-5.

Ribeiro, J.M., Zeidner, N.S., Ledin, K., Dolan, M.C. \& Mather, T.N. (2004) How much pilocarpine contaminates pilocarpineinduced tick saliva? Medical and Veterinary Entomology, 18, $20-4$.

Ryder, J.W., Pinger, R.R. \& Glancy, T. (1992) Inability of Ixodes cookei and Amblyomma americanum nymphs (Acari: Ixodidae) to transmit Borrelia burgdorferi. Journal of Medical Entomology, 29, 525-30.

Sanders, F.H. Jr \& Oliver, J.H. Jr (1995) Evaluation of Ixodes scapularis, Amblyomma americanum, and Dermacentor variabilis (Acari: Ixodidae) from Georgia as vectors of a Florida strain of the Lyme disease spirochete, Borrelia burgdorferi. Journal of Medical Entomology, 32, 402-6.

Sauer, J.R. \& Hair, J.A. (1972) The quantity of blood ingested by the lone star tick (Acarina: Ixodidae). Annals of the Entomological Society of America, 65, 1065-8.

Schulze, T.L. Lakat, M.F. Parkin, W.E. Shisler, J.K. Charette, D.J. \& Bosler, E.M. (1986) Comparison of rates of infection by the
Lyme disease spirochete in selected populations of Ixodes dammini and Amblyomma americanum (Acari: Ixodidae). Zentralblatt fur Bakteriologie, Mikrobiologie, und Hygiene Series A, 263, 72-8.

Sonenshine, D.E. (1991) Biology of Ticks, Vol. 1. Oxford University Press, New York, pp. 119-188.

Sonenshine, D.E., Ratzlaff, R.E., Troyer, J., Demmerle, S., Demmerle, E.R., Austin, W.E., Tan, S., Annis, B.A. \& Jenkins, S. (1995) Borrelia burgdorferi in eastern Virginia: comparison between a coastal and inland locality. American Journal of Tropical Medicine and Hygiene, 53, 123-33.

Stromdahl, E.Y., Evans, S.R., O'Brien, J.J. \& Gutierrez, A.G. (2001) Prevalence of infection in ticks submitted to the human tick test kit program of the U.S. Army Center for Health Promotion and Preventive Medicine. Journal of Medical Entomology, 38, 67-74.

Teltow, G.J., Fournier, P.V. \& Rawlings, J.A. (1991) Isolation of Borrelia burgdorferi from arthropods collected in Texas. American Journal of Tropical Medicine and Hygiene, 44, 469-74.

Valenzuela, J.G., Francischetti, I.M., Pham, V.M., Garfield, M.K., Mather, T.N. \& Ribeiro, J.M. (2002) Exploring the sialome of the tick Ixodes scapularis. Journal of Experimental Biology, 205, 2843-64.

Varela, A.S., Luttrell, M.P., Howerth, E.W., Moore, V.A., Davidson, W.R., Stalknecht, D.E. \& Little, S.E. (2004) First culture isolation of Borrelia lonestari, putative agent of southern tick-associated rash illness. Journal of Clinical Microbiology, 42, 1163-69.

Wikel, S.K. (1999) Tick modulation of host immunity: an important factor in pathogen transmission. International Journal of Parasitology, 29, 851-9.

Wolfinger, R. \& O'Connell, M. (1993) Generalized linear mixed models: a pseudo-likelihood approach. Journal of Statistical Computation and Simulation, 48, 223-43.

Zeidner, N.S., Schneider, B.S., Nuncio, M.S., Gern, L. \& Piesman, J. (2002) Coinoculation of Borrelia spp. with tick salivary gland lysate enhances spirochete load in mice and is tick species-specific. Journal of Parasitology, 88, 1276-8.

Zhu, K., Dillwith, J.W., Bowman, A.S. \& Sauer, J.R. (1997) Identification of hemolytic activity in saliva of the lone star tick (Acari: Ixodidae). Journal of Medical Entomology, 34, 160-6.

Accepted 10 December 2004 